\title{
Two-dimensional differential interference contrast microscopy based on four-hole variation of Young's interference
}

Matthew Lew, Xiquan Cui, Xin Heng, Changhuei Yang

Matthew Lew, Xiquan Cui, Xin Heng, Changhuei Yang, "Two-dimensional differential interference contrast microscopy based on four-hole variation of Young's interference," Proc. SPIE 6859, Imaging, Manipulation, and Analysis of Biomolecules, Cells, and Tissues VI, 685916 (29 February 2008); doi: $10.1117 / 12.760118$

SPIE. Event: SPIE BiOS, 2008, San Jose, California, United States 


\title{
Two-dimensional differential interference contrast microscopy based on four-hole variation of Young's interference
}

\author{
Matthew Lew*a, Xiquan Cui ${ }^{\mathrm{a}}$, Xin Heng ${ }^{\mathrm{a}}$, Changhuei Yang ${ }^{\mathrm{a}}$ \\ ${ }^{a}$ Dept. of Electrical Engineering, California Institute of Technology, MC 136-93, Pasadena, CA, \\ USA 91125
}

\begin{abstract}
We demonstrate a novel method of two-dimensional differential interference contrast (DIC) microscopy. Our method is cheaper, more compact, and more robust compared to conventional DIC microscopes; since it uses a simple variation of Young's double-slit geometry, no expensive or complex optical components are needed. In addition, our method quantitatively measures differential phase, unlike conventional DIC, which makes our device useful for optical metrology and cell biology applications. The device consists of four circular holes arranged in a "plus" pattern, milled into a metal layer $80 \mu \mathrm{m}$ above a complimentary metal-oxide semiconductor (CMOS) image sensor. Light incident upon the four-hole aperture is transmitted through the holes and creates an interference pattern on the CMOS sensor. This pattern shifts as a function of the spatial phase gradient of the incident light. By capturing the amplitude and location of the zero-order fringe of the interference pattern, the amplitude and differential phase of the incident light can be measured simultaneously. In this article, we model the response of the device using both geometric optics and Huygens principle. We then verify these models by experimentally measuring the responsivity of our device. A short analysis on the algorithm used to calculate the fringe location follows. We then show a beam profiling application by measuring the amplitude and spatial phase gradient of a Gaussian laser beam and an optical vortex. Finally, we show a DIC microscope application; we image a phase mask of the letters "CIT".
\end{abstract}

Keywords: differential interference contrast microscopy, interference, phase imaging

\section{INTRODUCTION}

A compact, high-resolution $(\sim 1 \mu \mathrm{m})$ phase imaging device would be a boon for applications such as phase microscopy, optical beam profiling, and optical wavefront sensing. Such a device would also provide cost savings if it can be produced using conventional microfabrication techniques and contain few or no optical components. A quick review of current phase imaging methods will reveal that many cannot be easily miniaturized without significant technical difficulties. For example, the Shack-Hartmann wavefront sensor ${ }^{1}$ utilizes microlenses that are costly to fabricate at scales of $\sim 1 \mu \mathrm{m}$. Similarly, phase contrast ${ }^{2}$ and differential interference contrast ${ }^{3}$ (DIC) microscopes use a complex configuration of optical components that are difficult to miniaturize.

We have shown previously ${ }^{4}$ that such a device can be created by modifying Young's double-slit geometry. A four-hole aperture is milled into a metal layer evaporated on top of an image sensor chip. The interference pattern of the aperture shifts with respect to the spatial phase gradient of the incident light. Therefore, the amplitude and differential phase of the incident light can be measured by the amplitude and location of the interference pattern on the image sensor. Thus, the amplitude and differential phase profile of an entire sample can be measured by raster scanning our device across the sample. It is important to note that the interference pattern of the four-hole aperture is surprisingly small ( $15 \times 15$ pixels). Therefore, it is possible to employ imaging methods such as $\mathrm{SHArP}^{5}$ to parallelize the imaging process. In this article, we analyze the responsivity of this device both theoretically and experimentally. We then use the device in an optical beam profiling application by measuring the amplitude and differential phase profile of an optical vortex. Finally, we image a phase mask of the letters "CIT", thereby demonstrating the DIC microscopy application of our device.

*mlew@its.caltech.edu; phone 626.395.4711; fax 626.395.8475; http://biophot.caltech.edu

Imaging, Manipulation, and Analysis of Biomolecules, Cells, and Tissues $\mathrm{VI}$, edited by Daniel L. Farkas, Dan V. Nicolau, Robert C. Leif,

Proc. of SPIE Vol. 6859, 685916, (2008) -1605-7422/08/\$18 · doi: 10.1117/12.760118

Proc. of SPIE Vol. $6859685916-1$ 


\section{MEDTHODOLOGY}

\subsection{Device geometry and fabrication parameters}

The geometry and operating principle of our device is shown in Figure 1. We begin with a 10-bit grayscale CMOS image sensor (Micron MT9V403) with $9.9 \mu \mathrm{m}$ x $9.9 \mu \mathrm{m}$ pixels. We then spin-coat an $80 \mu \mathrm{m}$-thick layer of transparent photoresist (SU-8) on top of the image sensor. Finally, we evaporate a $100 \mathrm{~nm}$-thick layer of silver on top of the SU-8 and use a focused ion beam (FEI Company Nova 200 Nanolab) to mill four-hole apertures into the metal layer. Two types of four-hole apertures were milled into the metal layer: one with $0.6 \mu \mathrm{m}$ diameter holes with $1.2 \mu \mathrm{m}$ spacing and the other with $1 \mu \mathrm{m}$ holes and $1 \mu \mathrm{m}$ spacing. Scanning electron microscope (SEM) images and the corresponding interference patterns of the apertures are shown in Figure 2.

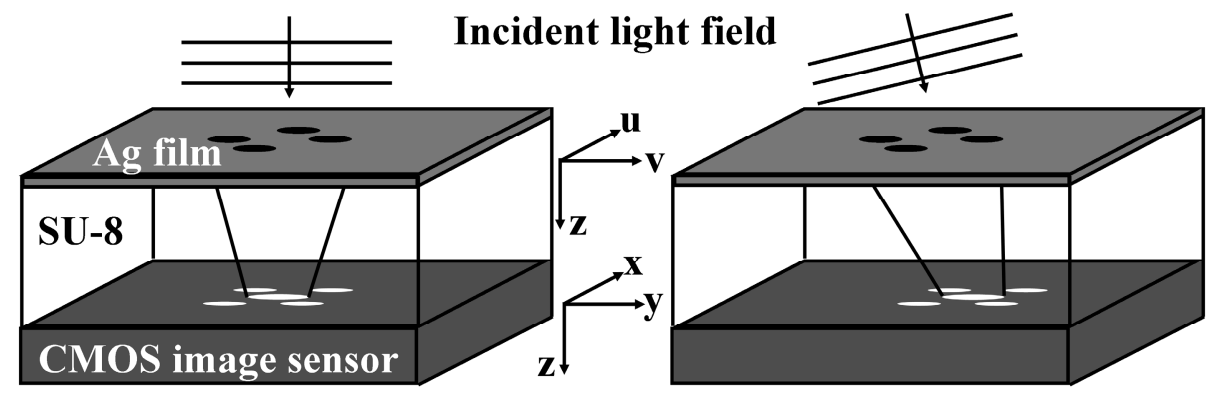

Fig. 1. Device geometry and principle of operation.
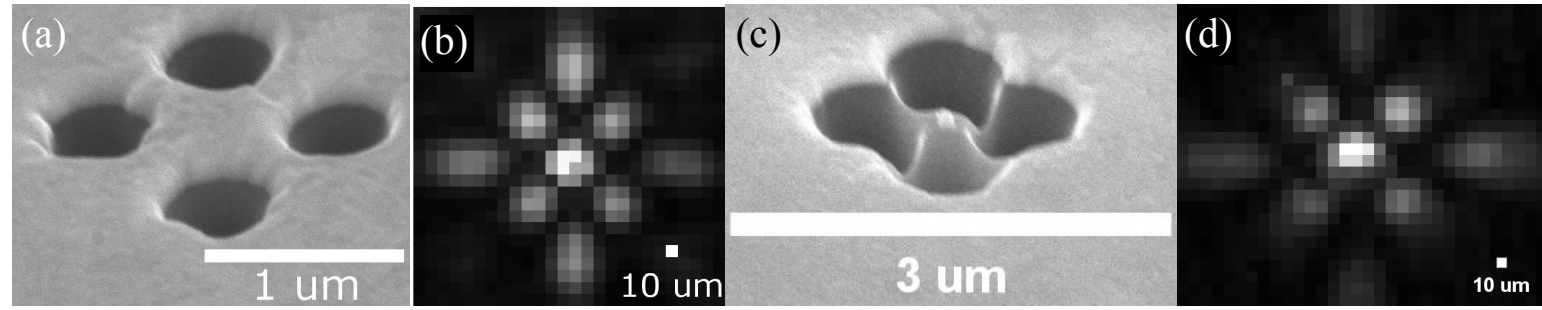

Fig. 2. (a) SEM image and (b) corresponding interference pattern of $0.6 \mu \mathrm{m}$ diameter holes with $1.2 \mu \mathrm{m}$ spacing. (c) SEM image and (d) corresponding interference pattern of $1 \mu \mathrm{m}$ holes with $1 \mu \mathrm{m}$ spacing.

\subsection{Theory of device operation}

Theoretically, the response of our device can be analyzed using geometrical optics, much like Young's double-slit experiment. Suppose there is an incident plane wave on our four-hole aperture. Let $d$ be the distance between the aperture and the CMOS image sensor, and let $x_{\text {zerofringe }}$ be the location of the zero-order interference fringe on the CMOS sensor (see Figure 1 for coordinate system definition). If the plane wave is normal to the aperture plane, then the zeroorder interference fringe will be located directly beneath the aperture, i.e. $x_{\text {zerofringe }}=0$. Now, suppose the plane wave is tilted so that it makes an angle $\theta$ in the $u z$ plane with respect to the normal. Assume that the transmission angle of the light through the four-hole aperture is also given by $\theta$. If $d$ is large with respect to $x_{\text {zerofringe, }}$ or equivalently if $\theta$ is small, we find that $x_{\text {zeroffinge }}$ is given by

$$
x_{\text {zerofringe }}=d \tan (\theta) .
$$

We now relate $\theta$ to the spatial phase gradient of the incident plane wave $\partial \phi(u, v) / d u$. Let $a$ be the spacing between holes in the $u$ and $v$ directions and $\lambda$ is the wavelength of the incident light. The phase difference $\Delta \phi$ of the light entering the holes along the $u$ axis is therefore given by

$$
\Delta \phi=\frac{2 \pi}{\lambda} a \sin (\theta)=\frac{\partial \phi(u, v)}{\partial u} a
$$

Rearranging Eq. (2) in terms of $\theta$ and substituting it into Eq. (1), we obtain 


$$
x_{\text {zerofringe }}=d \tan \left[\arcsin \left(\frac{\lambda}{2 \pi} \frac{\partial \phi(u, v)}{\partial u}\right)\right] .
$$

Notice that the responsivity does not depend on the hole spacing $a$. From Eq. (3), we can see that responsivity favors a large distance between the metal layer and the CMOS sensor. However, fringe intensity is inversely proportional to this distance, imposing a trade-off between responsivity and signal strength.

We verified Eq. (3) by simulating the response of our four-hole aperture device using Huygens principle. The four-hole aperture was represented as a two-dimensional array of point sources. To simulate a tilted plane wave in the $u z$ plane incident upon the four-hole aperture, the point sources were represented by the complex phase term $\exp (i \phi)$, where $\phi$ is given by

$$
\phi=\frac{\partial \phi(u, v)}{\partial u} u
$$

Summing the electric field contribution from each point source and taking the absolute value of this sum gives the intensity of the interference pattern in the imaging plane. The fringe location was computed by fitting the zero-order fringe to a two-dimensional Gaussian function (see section 2.4). These locations, and Eq. (3) for comparison, are plotted for various values of $\partial \phi(u, v) / \partial u$ in Figure 3.

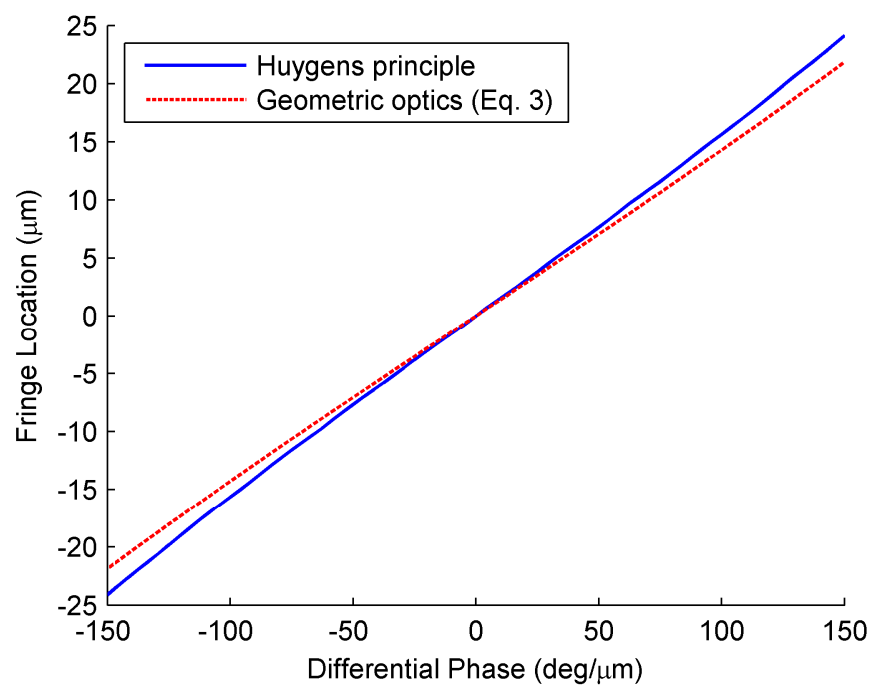

Fig. 3. Comparison of the predicted responsivity curve of the four-hole aperture using Huygens principle versus geometric optics.

\subsection{Experimental measurement of device response}

We experimentally verified Eq. (3) by illuminating our device with a collimated He-Ne laser beam (632.8 $\mathrm{nm}$ wavelength, $25.4 \mathrm{~mm}$ spot diameter, $20 \mathrm{~mW}$ intensity). We used goniometers to control the tilt of the device in the $u$ and $v$ directions, thereby varying the spatial phase gradient of the incident laser beam. The measured correlation between spatial phase gradient and fringe location is shown in Figure 4. The fringe location was computed by fitting the zeroorder fringe to a two-dimensional Gaussian function (see section 2.4). The measured correlation was fit to a linear curve; for our device parameters $(\lambda=632.8 \mathrm{~nm})$, Eq. (3) fits to linear curve with an $R$-square value of 0.995 for $|\partial \phi(u, v) / \partial u| \leq 360^{\circ} / \mu m$. Figure 4 shows that device responsivity does not depend on hole size or hole spacing, thereby verifying Eqn. (3). 

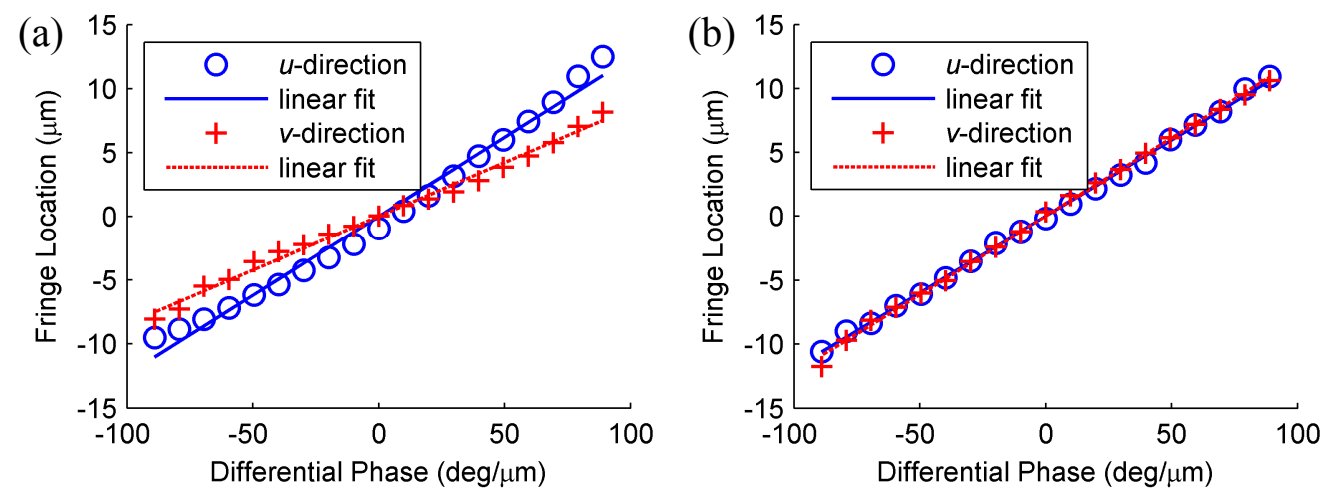

Fig. 4. Responsivity curves in the $u$ and $v$ directions for (a) $0.6 \mu \mathrm{m}$ holes with $1.2 \mu \mathrm{m}$ spacing and (b) $1 \mu \mathrm{m}$ holes with $1 \mu \mathrm{m}$ spacing.

\subsection{Computing the location of the zero-order interference pattern}

Two methods were utilized for finding the location of the zero-order interference fringe. The first algorithm is outlined below.

1. Find the pixel with the largest intensity value. This should roughly correspond to the center of the zero-order fringe. See Figure 5(a).

2. Use that pixel as the center of a row of five pixels. The values of this row of pixels are input into the MATLAB Gaussian curve-fitting function fit(), which fits the data to a one-dimensional Gaussian function. This function is characterized by a mean and standard deviation; the mean yields the center location of the fringe in the horizontal direction. See Figure 5(b).

3. Similar to step 2, use the pixel with the largest intensity value as the center of a column of five pixels. By fitting this column to a Gaussian function, we obtain the center location of the fringe in the vertical direction. See Figure 5(c).

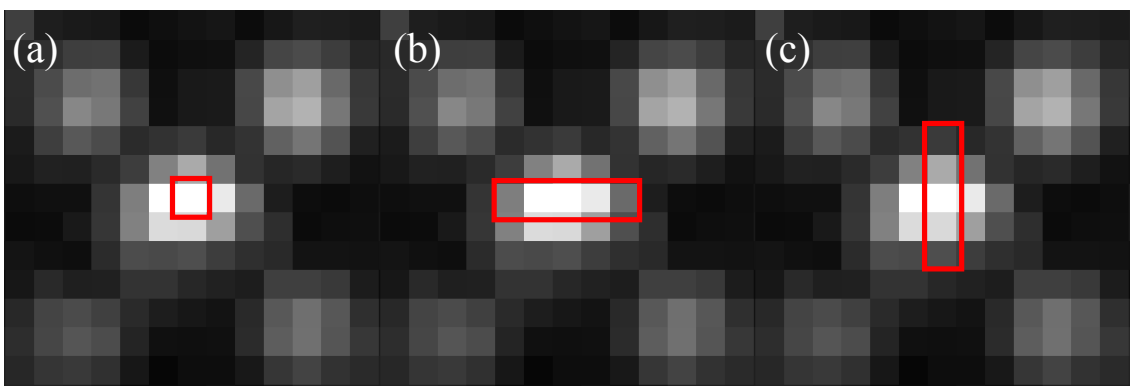

Fig. 5. (a) Finding the pixel with the largest intensity. (b) Choosing a row of five pixels to fit to a Gaussian function. (c) Choosing a column of five pixels to fit to a Gaussian function.

As is apparent from Figure 5, not all of the zero-order fringe data is used to find the center location of the fringe, which is a serious weakness of this algorithm. Furthermore, the performance of the algorithm degrades as the true location of the fringe moves toward pixel boundaries.

The current algorithm used to find the center location of the zero-order interference fringe uses unconstrained nonlinear optimization (MATLAB function fminsearch ()). The optimization algorithm minimizes the mean-squared error between the interference pattern and a two-dimensional Gaussian function, $u(x, y)$, where

$$
u(x, y)=A \exp \left[-\left(\frac{x-\mu_{x}}{\sigma_{x}}\right)^{2}\right] \exp \left[-\left(\frac{y-\mu_{y}}{\sigma_{y}}\right)^{2}\right] .
$$


The parameters $A, \mu_{x}, \mu_{y}, \sigma_{x}$, and $\sigma_{y}$ yield five degrees of freedom, and we use the optimized $\mu_{x}$ and $\mu_{y}$ as the location of the zero-order fringe. The optimized $A$ is proportional to the intensity of the fringe.

The superiority of the two-dimensional Gaussian fit over the two one-dimensional Gaussian fits is shown in Figure 6. The two-dimensional fit yields a correlation between fringe location and differential phase that is much more linear than the correlation given by the two one-dimensional fits. The data used for these fits is the same data used for Figure 4(b).
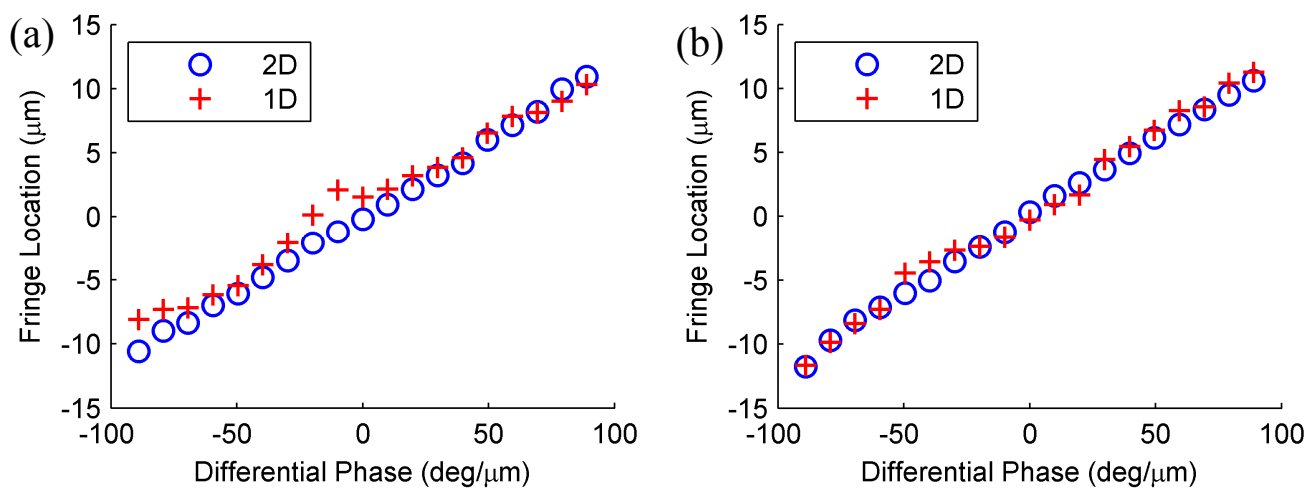

Fig. 6. Contrasting the performance of the two-dimensional Gaussian curve-fitting algorithm versus the one-dimensional Gaussian curve-fitting algorithm for finding the correlation between fringe location and differential phase in the (a) $u$ direction and (b) $v$ direction.

\subsection{Creating a computer generated hologram for an optical vortex}

To demonstrate a beam-profiling application of our device, we used a computer generated hologram to create an optical vortex. ${ }^{6}$ An optical vortex is a helical mode of light whose phase is given by $\exp (\operatorname{im} \theta)$, where $\theta$ is the polar angle about the beam's axis and $m$ is the topological charge of the vortex. ${ }^{7}$ A phase singularity exists on the beam's optical axis, causing a null in the optical vortex's intensity profile. The differential phase profile of the optical vortex is orthogonal to that of a Gaussian beam, making the optical vortex an ideal complementary beam profile to the Gaussian beam for testing our device.

In order to create the hologram, we computed the interference pattern of a plane wave (reference beam) and an optical vortex (object beam). We represent the amplitudes of the electric fields of these beams in the hologram plane as

$$
\begin{gathered}
E_{\text {plane }}(x, y)=\exp \left(i \frac{2 \pi x \sin (\psi)}{\lambda}\right) \text { and } \\
E_{\text {vortex }}(r, \theta)=\exp (i m \theta),
\end{gathered}
$$

where $\psi$ is the angle between the wave vector of the plane wave and the wave vector of the optical vortex, $\lambda$ is the wavelength of the reference beam, and $m$ is the topological charge of the vortex. The interference pattern is then given by

$$
\begin{aligned}
I(x, \theta) & =\left|E_{\text {plane }}+E_{\text {vortex }}\right|^{2} \\
& =2\left[1+\cos \left(\frac{2 \pi x \sin (\psi)}{\lambda}+m \theta\right)\right] .
\end{aligned}
$$

Eq. (8) shows that the grating period decreases with increasing $\psi$. If the grating period is too low, the quality of the hologram will suffer due to limited fabrication resolution; therefore, $\psi$ was chosen to be two degrees so that there was an acceptable compromise between grating period and beam separation.

In order to fabricate a chrome mask with the desired interference pattern, the continuous intensity distribution (grayscale) given by Eq. (8) must be converted to a binary intensity distribution (black and white). Figure 7 compares the continuous interference pattern to the converted binary intensity pattern. We computed several holograms with the 
parameters $\psi=2$ degrees, $\lambda=632.8 \mathrm{~nm}$, and $m=\{1,2,3\}$. We then fabricated a chrome mask (100 nm thickness) with these interference patterns.
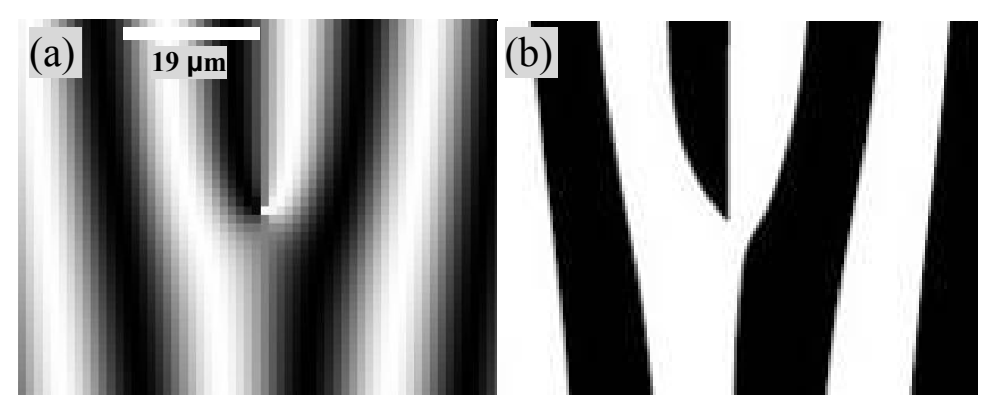

Fig. 7. (a) Computer generated hologram showing the interference pattern of a plane wave and an optical vortex $(m=1)$ at a two-degree incidence angle for $\lambda=632.8 \mathrm{~nm}$. (b) Binary conversion of hologram in (a) for chrome mask printing.

\section{RESULTS}

\subsection{Imaging a Gaussian beam}

We first used our device to measure the amplitude and differential phase of a Gaussian laser beam. A collimated He-Ne laser beam (4.2 $\mathrm{mm}$ spot diameter, $2 \mathrm{~mW}$ intensity) was focused ahead of our device (i.e. $z>0$ in our device plane) using an aspheric lens (Thorlabs C220TME-B, $11 \mathrm{~mm}$ focal length). This yields a differential phase pattern given by

$$
\begin{aligned}
\nabla \phi_{\text {Gaussian }}(u, v, z) & =\frac{\partial \phi_{\text {Gaussian }}(u, v, z)}{\partial u} \hat{u}+\frac{\partial \phi_{\text {Gaussian }}(u, v, z)}{\partial v} \hat{v}, \\
& =\frac{k u}{z\left[1+\left(z_{0} / z\right)^{2}\right]} \hat{u}+\frac{k v}{z\left[1+\left(z_{0} / z\right)^{2}\right]} \hat{v},
\end{aligned},
$$

where $k=2 \pi / \lambda, z_{0}=\pi w_{0}^{2} / \lambda$, and $w_{0}$ is the minimum waist radius of the beam. Our device was raster-scanned across the beam by motorized translation stages (Newport CMA-25CCCL) powered by a Newport ESP-300 motor controller. The motor controller and camera were controlled by custom software to coordinate the scanning and image capture operations. The amplitude and differential phase patterns of the Gaussian beam are shown in Figure 8. Note that crosstalk between Figures 8(a), 8(b), and 8(c) is virtually nonexistent. Therefore, our device does not need a uniform intensity distribution in order to accurately measure differential phase.
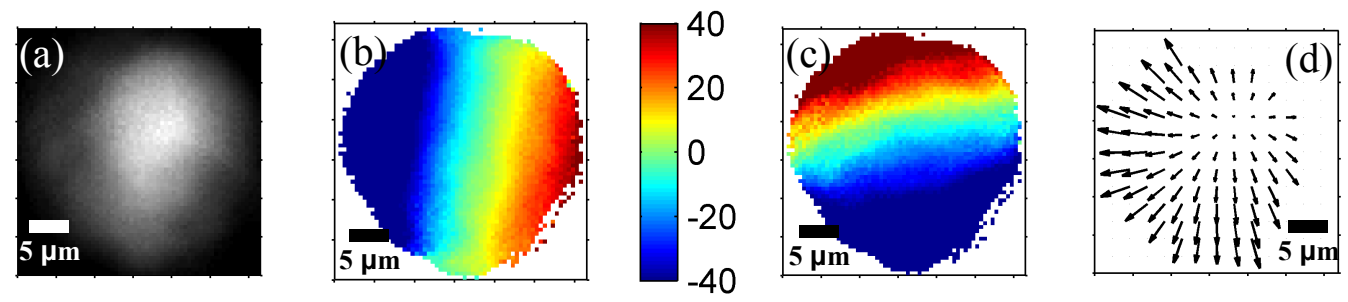

Fig. 8. (a) Intensity, (b) differential phase in the $u$ direction, (c) differential phase in the $v$ direction, and (d) vector representation of the differential phase of a Gaussian laser beam. Colorbar is in units of degrees/micrometer.

\subsection{Imaging an optical vortex}

The interference pattern of an optical vortex of topological charge one and a plane wave intersecting at a 2-degree incidence angle was computed and patterned onto a chrome mask (see section 2.5). A collimated He-Ne laser beam (2 $\mathrm{mW}$ intensity) was projected onto the hologram, and the first-order diffraction component was focused (i.e. $z=0$ in our device plane) onto the four-hole aperture with $0.6 \mu \mathrm{m}$ holes and $1.2 \mu \mathrm{m}$ spacing with a plano-convex lens (Thorlabs LA1951, $25.4 \mathrm{~mm}$ focal length). The differential phase profile of an optical vortex is given by 


$$
\Delta \phi_{\text {vortex }}(u, v, z=0)=-\frac{m v}{u^{2}+v^{2}} \hat{u}+\frac{m u}{u^{2}+v^{2}} \hat{v},
$$

where $m$ is a signed integer called the topological charge, or winding number, of the optical vortex. The four-hole aperture was scanned across the vortex to measure its amplitude and differential phase profiles, which are shown in Figure 9. The differential phase of the vortex rotates about the optical axis and is larger in magnitude near its center, as predicted by Eq. (5).
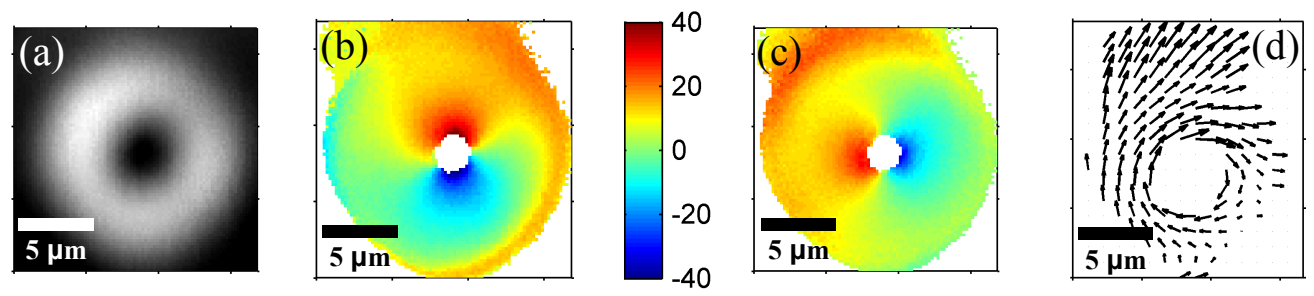

Fig. 9. (a) Intensity, (b) differential phase in the $u$ direction, (c) differential phase in the $v$ direction, and (d) vector representation of the differential phase of an optical vortex. Colorbar is in units of degrees/micrometer.

\subsection{Imaging a phase mask}

To test the DIC microscopy application of our device, we imaged a phase mask of the letters "CIT". The phase mask consisted of a glass coverslide uniformly coated with polymethyl methacrylate (PMMA). Electron beam lithography was then used to etch the letters "CIT" $100 \mathrm{~nm}$ deep into the PMMA. Since the materials of this phase mask are transparent, these letters exhibit poor contrast under bright-field microscopy but are easily revealed under DIC microscopy.

We illuminated the phase mask with a halogen lamp and raster-scanned our device across a portion of the "T". The intensity and differential phase images of the phase mask are shown in Figure 10. Note how the contrast of the intensity image is poor; most of the features in this image are due to scattering of the light at the boundaries of the "T." However, the differential phase images show good contrast at the edges of the "T," where the change in optical path length is largest.
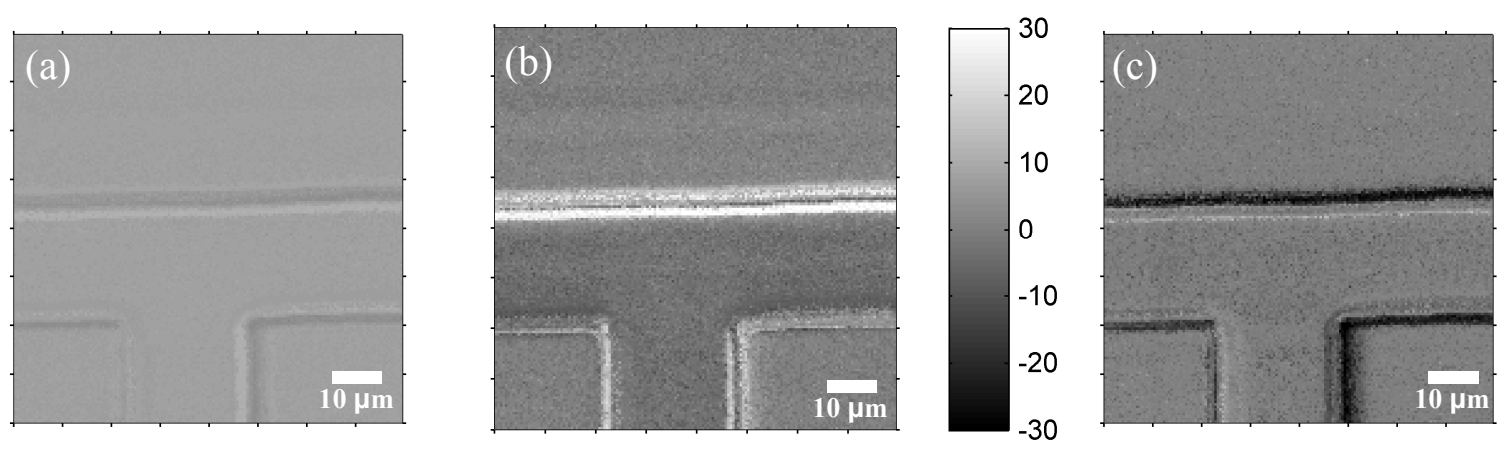

Fig. 10. (a) Intensity image, (b) differential phase image in the $u$ direction, and (c) differential phase image in the $v$ direction of a "T" phase mask. Colorbar is in units of degrees/micrometer.

\section{CONCLUSIONS}

In conclusion, we have demonstrated a novel device for high-resolution phase imaging that utilizes a four-hole aperture similar to Young's double slit geometry. The device uses the intensity and location of the interference pattern of the four-hole aperture to measure the amplitude and differential phase of the incident optical wavefront. This device has the advantages of being compact and lensless; it is therefore an excellent alternative to conventional wavefront sensing methods such as the Shack-Hartmann wavefront sensor and phase imaging methods such as phase microscopy and DIC microscopy. In particular, we have shown the viability of this device for both optical beam profiling and DIC microscopy applications. While the single-aperture nature of this device requires it to be scanned across samples in order 
to image them, the scanning process is easily parallelizable. Since the number of sensor pixels required to capture the interference pattern of the four-hole aperture is small, a slanted one-dimensional array or two-dimensional array of fourhole apertures can be used to parallelize the scanning process, much like SHArP. ${ }^{5}$ Furthermore, a microfluidic channel can be fabricated on top of this array of apertures to efficiently scan biological cells, creating a phase-sensitive optofluidic microscope. ${ }^{8}$ One weakness of our device is that it is intrinsically lossy since we are sampling the light field

of interest with small holes. This was not an issue in our experiments, but using stronger illumination or more sensitive imaging sensors can compensate for this loss.

\section{ACKNOWLEDGEMENTS}

The authors acknowledge financial support from the Defense Advanced Research Projects Agency Center for Optofluidic Integration. We thank S. Han, J. Wu, and $\mathrm{Z}$. Yaqoob for their technical assistance and enlightening discussions.

\section{REFERENCES}

1 J. Z. Liang, B. Grimm, S. Goelz et al., "Objective measurement of wave aberrations of the human eye with the use of a Hartmann-Shack wave-front sensor," Journal of the Optical Society of America a-Optics Image Science and Vision 11 (7), 1949-1957 (1994).

2 M. Pluta, "Stray-light problem in phase contrast microscopy or toward highly sensitive phase contrast devices: a review," Optical Engineering 32 (12), 3199-3214 (1993).

3 M. R. Arnison, K. G. Larkin, C. J. R. Sheppard et al., "Linear phase imaging using differential interference contrast microscopy," Journal of Microscopy-Oxford 214, 7-12 (2004).

4 M. Lew, X. Q. Cui, X. Heng et al., "Interference of a four-hole aperture for on-chip quantitative two-dimensional differential phase imaging," Optics Letters 32 (20), 2963-2965 (2007).

5 X. Q. Cui, X. Heng, J. G. Wu et al., "Slanted hole array beam profiler (SHArP)- a high-resolution portable beam profiler based on a linear aperture array," Optics Letters 31 (21), 3161-3163 (2006).

6 Z. S. Sacks, D. Rozas, and G. A. Swartzlander, "Holographic formation of optical-vortex filaments," Journal of the Optical Society of America B-Optical Physics 15 (8), 2226-2234 (1998).

7 J. E. Curtis and D. G. Grier, "Structure of optical vortices," Physical Review Letters 90 (13) (2003).

8 X. Heng, D. Erickson, L. R. Baugh et al., "Optofluidic microscopy - a method for implementing a high resolution optical microscope on a chip," Lab on a Chip 6 (10), 1274-1276 (2006). 